\title{
Physical activity in secondary stroke prevention
}

\section{EXERCISE IS MEDICINE / NEUROLOGY / PA PROMOTION}

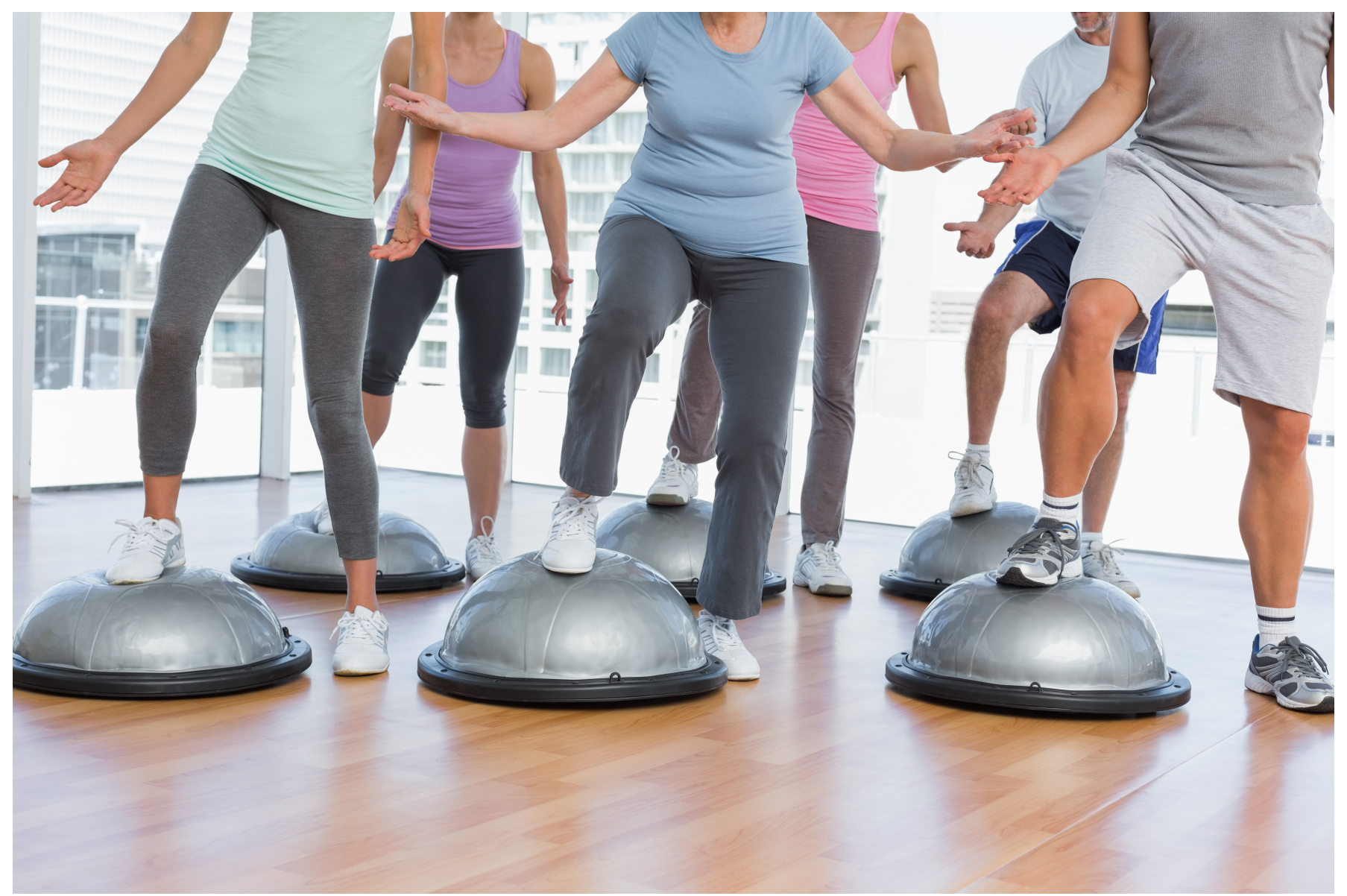

Scutelnic A, Arnold M, Jung S, Heldner MR

Department of Neurology, Inselspital, University Hospital and University of Bern, Bern, Switzerland

\section{Summary}

After having a stroke the main challenges are reducing the risk of recurrent stroke, improving impaired brain function, quality of life, independence in activities of daily living and reintegration into the community. [1] Lesion-induced impairment of brain function also has, besides its effects on e.g. motor, sensory, visual and speech function, an influence on e.g. cognition and mood, all of which are determinants of post-stroke physical activity.

The evidence for a benefit of physical activity in secondary stroke prevention is increasing and treatment strategies aimed at factors which are limiting physical activity are more and more recognized. 


\section{Zusammenfassung}

Die grössten Herausforderungen nach einem Hirnschlag sind die Reduktion des Risikos eines erneuten Hirnschlags, der Konsequenzen und das Verbessern der beeinträchtigten Hirnfunktion, der Lebensqualität, der Selbstständigkeit im Alltag und die Reintegration in der Gesellschaft. [1] Die durch den Hirnschlag eingeschränkte Hirnfunktion kann nebst mit motorischen, sensiblen, visuellen und sprachlichen Einschränkungen auch mit Störungen der Kognition und der Stimmung einhergehen. Diese Defizite wirken sich auf die körperliche Aktivität nach einem Hirnschlag aus.

Der günstige Effekt der körperlichen Aktivität in der Sekundärprävention des Hirnschlags ist immer mehr belegt, und Behandlungsstrategien hinsichtlich der die körperliche Aktivität limitierenden Faktoren werden zusehends als wirksam anerkannt.

\section{Résumé}

Après un accident vasculaire cérébral (abr. AVC) les plus grands défis sont la réduction de risque d'un nouveaux AVC, des conséquences d'AVC, l'amélioration des fonctions perdues, de la qualité de vie, de l'indépendance dans les activités journalières et de la réintégration sociale. [1] L'AVC peut affecter autant la fonction moteur, sensible, visuelle et la langue que la cognition et l'humeur. Ces fonctions représentent des facteurs limitants pour l'activité physique après l'AVC.

L'évidence pour un effet bénéfique de l'activité physique après l'AVC devient de plus en plus consistante et des stratégies pour améliorer les facteurs limitants pour l'activité physique sont de plus en plus reconnu comme efficace.

\section{Case report}

A 65-year old female patient suffered an acute ischaemic stroke with acute right sided hemineglect, global aphasia and right sided hemiplegia, corresponding to a NIHSS score of 15 points. The head MRarteriography showed an acute ischaemic lesion as depicted in Figure 1. Successful bridging- therapy was performed. Head MR-arteriography 24 hours after thrombectomy showed open vessels and no new ischaemic lesion. So, the patient was mobilized to the edge of the bed on day 1 . In cardiac rhythm monitoring, an episode of atrial fibrillation of 90 seconds duration occurred. Rivaroxaban $20 \mathrm{mg}$ daily was initiated, as was a high-dose statin (LDL-cholesterol levels of $4.8 \mathrm{mmol} / \mathrm{L}$ ) and an ACE-inhibitor because of newly diagnosed arterial hypertension.

At discharge, a mild non-fluent aphasia and a moderate right sided hemiparesis persisted, corresponding to a NIHSS score of 4 points. The patient got transferred to a rehabilitation clinic, were a supervised 3weeks physical therapy programme was started.

After leaving the rehabilitation clinic, a spastic gait pattern with circumduction of the right leg persisted, which allowed for a 6-Minutes Walk Test distance of 177 meters. An ambulatory rehabilitation groupprogramme consisting of walking and stationary cycle ergometry followed, with $3 \mathrm{x}$ sessions/week of 30 minutes, at an intensity of $40-70 \%$ of age adjusted maximal heart rate. At three months a light residual gait impairment persisted, lightly affecting the activities of daily living. The 6-meter walk distance improved to 360 meters. 


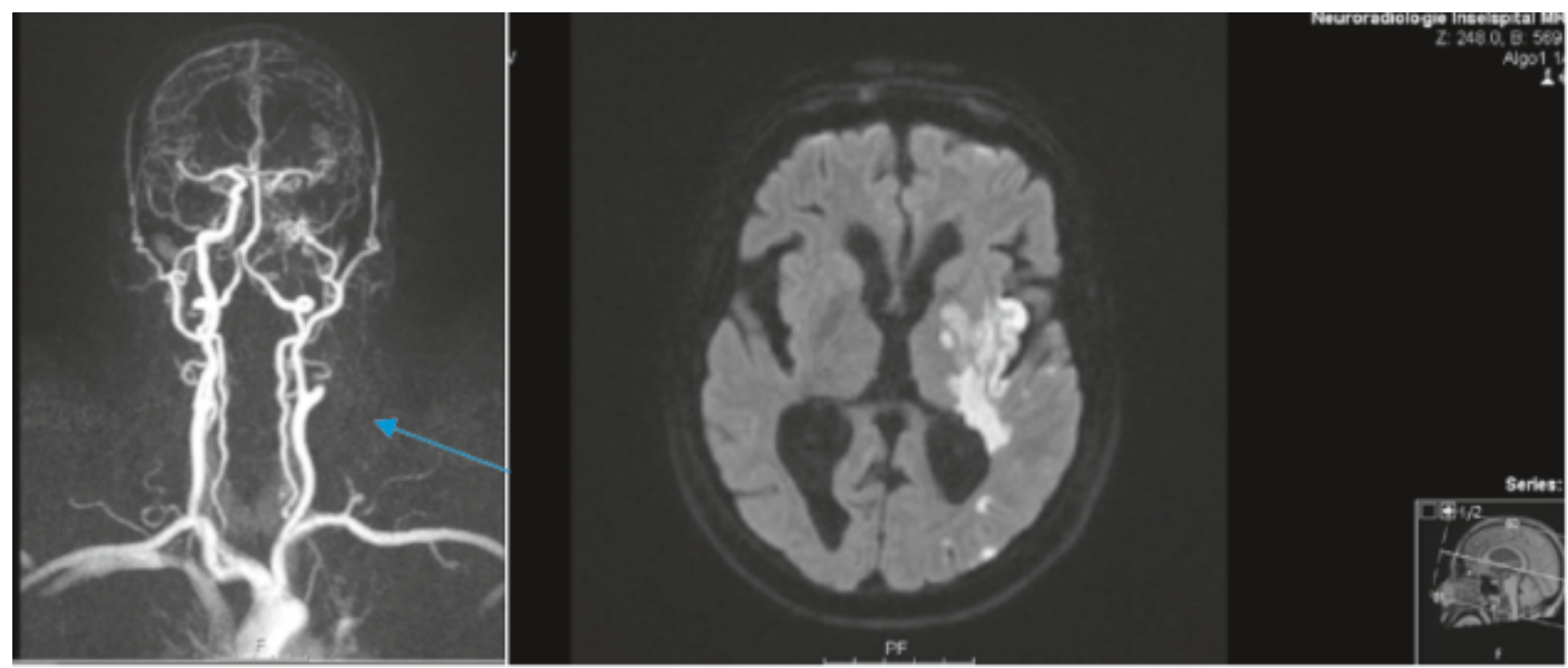

Figure 1: MR-arteriography und Diffusion-weighted-imaging sequences showing an acute ischaemic stroke in the territory of the left middle cerebral artery because of a left sided internal carotid artery occlusion (blue arrow).

\section{Introduction}

A recurrent stroke is potentially disabling, impairing activities of daily living, of mental health, decreasing quality of life and causing death. The risk of recurrent stroke but also of other vascular events after a first ever stroke is considerable. $[2,3,4,5]$ It can be lowered by timely identification of stroke aetiology and consecutive initiation of secondary prevention measures. [1,6]

However, although widely available, up to $60 \%$ of patients in Europe have been shown not to have access to basic interventions such as to cardiac monitoring, statins, blood-pressure control at follow-up, carotid endarterectomy, face-to-face follow-up in hospital, direct oral anticoagulants and to lifestyle management programmes. [3]

Also, there has been demonstrated to be impaired awareness and control of risk factors, including physical activity, after stroke. [3,7] An Austrian observational cohort study $(n=1,730)$ including $20.1 \%(n=348)$ participants with recurrent ischaemic stroke or transient ischaemic attack (abr. TIA) showed significantly poorer risk factor control, including lifestyle modification, compared with those with a first ever event. [7] A Danish observational study $(\mathrm{n}=121)$ found awareness of risk factors to be present at discharge from a stroke unit. 12 months after stroke there was an increase of proportion of inactive patients from 36 to $59 \%$ $(p<0.0001)$. Also, poor control of arterial hypertension, of cholesterol levels and unchanged cigarette smoking frequency, but less excessive alcohol consumption was found. [8]

Because of lesion-induced disruption of brain function, patients after stroke are showing a different behavior of physical activity. For example, there might be a reduced ability of the body to be physically active because of motor and sensory deprivation, impaired balance, falls and a decreased cardiorespiratory fitness. [9] Secondary depression, anxiety, pain, cognitive disturbances and fatigue are also affecting physical activity, as is impaired self-efficacy, which is the belief of being able to successfully complete a task. [10] 


\section{Physical activity after stroke}

A case-control study ( $n=13,249,39,747$ controls) demonstrated that stroke survivors are less likely to reach $150 \mathrm{~min} /$ week of physical activity compared to controls. [11]

A prospective study $(n=40)$ investigated physical activity in stroke survivors with accelerometers (which are measuring mobility, step number and calculating calories). Despite the ability to walk, some patients did not reach the daily recommended level of physical activity. Latter was defined as 30 minutes of exercise a day with an intensity of more than $142 \mathrm{kcal} / \mathrm{day}(1,000 \mathrm{kcal} / \mathrm{week})$ reaching 215-285 kcal/day (1,500-2,000 kcal/week). [12]

What are the factors that negatively affect physical activity after stroke? A recent meta-analysis of cohort and prospective cross-sectional studies ( $n=26,1,349$ stroke survivors) investigated factors associated with physical activity after stroke. It found higher age, female sex, impaired physical function (quantified with 6-Meter Walking Test distance, comfortable gait speed, Berg-BalanceScale and cardiorespiratory fitness), fatigue, depression, falls, impaired self-efficacy, balance and impaired health-related quality of life to be significantly associated with physical activity, whereas analyses for neglect, infarct side, cognition and time delay since stroke were inconclusive. Cognition measured with MoCA (Montreal cognitive assessment) - not with Mini Mental Test - showed significant association with physical activity. Moreover, physical function accounted for only half of the variance in post-stroke physical activity levels (defined as the quantity of the physical activity performed). [9]

\section{Self-efficacy}

Confidence is a measure of self-efficacy. Improving it, is one of 10 main priorities related to life after stroke, established by a consensus as agreed by stroke survivors, caregivers and health professionals. $[13,14]$

A systematic review, which included 17 studies showed a positive association between self-efficacy and mobility, activities of daily living, depression and health-related quality of life. [10]

Different self-efficacy enhancing strategies have been proposed [12] and studied prospectively in 4 randomized clinical trials (abr. RCTs) ( $\mathrm{n}=630$ participants). They have shown a trend to be effective for e.g. functional independency, walking and anxiety. [10]

\section{Fatigue}

Fatigue has a negative effect on physical activity on its own and by its contribution on health-related quality of life. [9,15]

Fatigue in stroke patients is defined as a feeling of early exhaustion developing during mental activity, with weariness, lack of energy and aversion to effort. [16] More than one type of fatigue in stroke patients has been proposed: chronic fatigue, which may be classified into primary (i.e. absence of any causes besides the stroke) or into secondary (i.e. caused by co-morbidities or use of certain medications) and activity-dependent fatigability or exertional fatigue, which is an activity-induced decrement in performance. $[1,17,18]$

Fatigue is prevalent after stroke. A survey-study in stroke survivors ( $n=4,023$, follow-up 2 years) concluded that $10 \%$ of patients always and $29 \%$ often were affected. [19] 
Chen et al. studied the correlation between post-stroke fatigue and health-related quality of life ( $\mathrm{n}=218$ patients) at 3 months after ischaemic stroke and concluded that severity of fatigue was a significant contributor to stroke-specific quality of life, accounting for $39 \%$ of the variance. [15] RCTs specifically studying the effect of physical activity alone on post-stroke fatigue are lacking. However, one RCT ( $n=83$ stroke patients) compared the effect of cognitive therapy combined with physical activity training on fatigue over 12 weeks. It showed that both treatment options had a significant benefit on fatigue, with the group of combined treatment having more gains on outcome scales (measuring fatigue, anxiety, depression, sickness, pain, sleep and walking). [20]

A systematic review identified three cross-sectional studies ( $n=444$ patients), which found no significant association of fatigue with physical activity. [17] However, one of those studies found an association of severe fatigue with low self-efficacy, causing less engagement in physical activity. The American Heart Association/American Stroke Association (AHA/ASA) Guidelines from 2014 recommend physical activity as a non-pharmacologic therapy for fatigue after stroke. [1]

An aggravating factor for fatigue is sleep-disordered breathing, which is prevalent after stroke. A systematic review ( $n=37$ studies, $n=3,242$ participants with cerebrovascular disease) demonstrated that obstructive sleep apnea, defined as an apnoe-hypopnoe index (AHI) >10 per hour in this study, was present in $61.9 \%$ of stroke survivors, mostly diagnosed 7-28 days after index-event. Identification and treatment of sleep apnea potentially has a beneficial effect on fatigue and possibly on physical activity behavior. [21] Another factor associated with fatigue in stroke survivors is post-stroke pain. Choi-Kwon et al. ( $\mathrm{n}=364$ patients, 1 year after ischaemic stroke) found that 135 (37.1\%) patients had pain, of which 78 (21.4\%) was musculoskeletal, 22 (6\%) central post-stroke type, 16 (4.4\%) combined and 19 (5.2\%) other pain. Pain was significantly associated with fatigue $(p=0.026)$ and $m R S(p=0.004)$. [22] The causality between pain and fatigue is unclear and may be bilateral. Identifying pain and treating it may have a positive effect on physical activity. Data regarding the direct effect of physical activity on post-stroke pain are lacking.

\section{Depression}

Depression is highly prevalent in stroke survivors and negatively associated with physical activity after stroke. [9]

After analyzing the data available from 51 publications ( $\mathrm{n}=25,671$ patients), Hackett et al. found depression among stroke survivors in 33\% (95\%CI 29-36\%). [23]

A retrospective cohort-study showed that post-stroke depression significantly increases disability. Effectiveness of rehabilitative treatment, activities of daily living and mobility was worse in patients with post-stroke depression, besides their longer length of hospital stay. Post-stroke depression added $15 \%$ to the disability in stroke survivors. [24]

A meta-analysis ( $n=1,022,13$ studies, data up to 2014 included) showed a significant reduction of depressive symptoms after resistance training, functional training, aerobic physical activity, treadmill physical activity, Bobath physical activity, individualized physical activity with education and community based rehabilitation services ( $p=0.03$ ). Physical activity had a positive effect on depressive symptoms across both the subacute ( $\leq 6$ months post-stroke) and chronic stage of recovery ( $>6$ months). Furthermore, there was a significant effect of physical activity on depressive symptoms when higher intensity studies were pooled. [25] 


\section{Cognitive impairment}

Cognitive impairment is common after stroke and cognitively impaired individuals are less likely to engage in physical activity. [26]

After a 5-year follow-up of 2,914 stroke survivors, aged over 65 years of age, $64 \%$ had cognitive impairment and patients with cognitive impairment were 6 times more likely to suffer a recurrent stroke than patients with normal cognition. Incident stroke was associated with transition from cognitive impairment no dementia to dementia. Cognitive impairment may be an independent risk factor for stroke through worse adherence to treatment and altered attitude of physicians towards control of risk factors. [27] There is evidence for a reverse causality between physical activity and cognition in stroke survivors. A systematic review (9 studies, up to 2011, n=716 participants) showed a significant benefit of physical activity on cognition ( $\mathrm{p}=0.015)$. [28]

\section{Falls}

Falls occur regularly in stroke survivors. Falls cause low self-efficacy, which is negatively associated with physical activity. [9]

In one study, between 14-65\% of people with stroke fell at least once during hospitalization and between $37-73 \%$ during the first 6 months. [29]

A RCT studied the effects of body-weight supported treadmill rehabilitation after stroke over 12-16 weeks in 408 stroke survivors (one group started the therapy 2 months after stroke, the 2nd group after 6 months) compared with a 3rd group (supervised home-exercise programme consisting of enhancing flexibility, range of motion in joints, strength of arms and legs, coordination and static and dynamic balance). No significant difference of falls was noted between the three groups. However, in the subgroup with severe walking impairment, early therapy showed more falls than late therapy ( $52 \%$ vs. $36 \%, p=0.05)$ or than the home group ( $52 \%$ vs. $30.3 \%$, $\mathrm{p}=0.009$ ). The balance however, showed a clinically relevant improvement. [30]

Another RCT ( $\mathrm{n}=61$ stroke patients, aged $>65$ years) assigned patients to two exercise programmes: an agility programme consisting of standing in various postures and walking with various challenges and a stretching/weight-shifting exercise programme focused on slow and low impact movements. The participants in the agility programme had significantly fewer falls than the stretching/weight-shifting programme. Functional balance was significantly improved with both interventions. [31]

\section{Miscellaneous factors}

The kinetic gait pattern in health controls is different from stroke survivors. Latter use other kinetic strategies to achieve an efficient walking. When applied to persons with stroke, movement patterns of healthy individuals do not translate into better results and trying to teach stroke patients to walk as healthy individuals do, may be counterproductive. [32] A systematic review ( $n=764,29$ studies) concluded that stroke survivors use more energy for comfortable walking speed than their healthy peers. [33] Physical activity preference changes after stroke were examined in another survey study ( $\mathrm{n}=23$ adult stroke survivors, $n=41$ controls). The study identified stroke survivors to prefer physical activity to be demonstrated to them and to be performed in a facility. [34] However, not all stroke patients have access 
to instructors and facilities.

Logistical difficulties are another factor negatively influencing the physical activity after stroke. Marzolini et al. $(n=61)$ studied the main factors related to poor attendance to an ambulatory stroke rehabilitation programme. Weather, transportation problems, health problems (e.g. hospital readmissions, influenza/colds, diabetes mellitus, cardiac complications and musculoskeletal issues), traveling long distances and language barrier were the main factors identified to negatively affect the attendance to ambulatory rehabilitation. [35]

Reduced cardiorespiratory fitness is another factor limiting physical activity after stroke. Cardiorespiratory fitness is the capacity of the heart, lungs, vessels and muscles to deliver oxygen and to remove metabolic products during physical activity. It reflects vascular health. [36]

Mackay-Lyon et al. measured VO2max maximal oxygen consumption, reflects the overall cardiorespiratory fitness) in stroke survivors ( $n=29)$ with unilateral deficits after 26+/-8.8 days and found that it corresponded to $60+/-16 \%$ of that of normative values for healthy sedentary individuals. [37] Physiological factors contributing to reduced cardiorespiratory fitness are: change of paretic-muscle structure, increased intramuscular fat and shift to fast-twitching fibers which are more insulin resistant and fatigue prone, increased muscle TNA-alfa, which is involved in muscle atrophy and insulin resistance, increased systemic pro-inflammatory cytokines (TNF-alfa, VCAM-1 and IL-6), altered glucose metabolism, impaired autonomic control of the vascular system not related to medication (e.g. beta-blockers) and impaired breathing mechanics because of muscle weakness. [36] A meta-analysis (17 studies, $n=4,571$ mostly ischaemic stroke survivors) showed a significant increase of cardiorespiratory fitness (measured in VO2max) and significantly better results in 6-Meter Walking Test with high intensity training (defined as achieving $>60 \%$ of heart rate reserve/VO2max or $>70 \% \mathrm{HRmax}$ ), compared to mild-to-moderate intensity training without increasing the rate of adverse events. [38]

\section{Effects of physical activity on risk factors and stroke risk in secondary stroke}

\section{prevention}

The question arises whether performing physical activity is having a positive effect on risk factors and lowering stroke and vascular recurrence rate. There is increasing evidence. After analyzing 12 meta-analyses ( $n=210,926$ patients, 106 studies) on secondary stroke prevention (11 meta-analyses) and mixed secondary/high risk primary prevention (1 meta-analysis) Hackam et al. concluded that 5 treatment interventions consisting of lifestyle modification, aspirin, a statin and an antihypertensive agent are sufficient to reduce the risk of a major vascular event (i.e. nonfatal stroke, nonfatal myocardial infarction, and vascular death) by $80 \%$, which would translate in a number needed to treat of 5 and in a residual 5-year risk of 5\%. [6] 11 trials included in the 12 meta-analyses studied physical activity $(n=2,285)$ with a relative risk reduction of a major vascular event (i.e. nonfatal stroke, nonfatal myocardial infarction, and vascular death) of $28 \%$ (relative risk 0.72, 95\%CI 0.54-0.95\%). Putting it into perspective, Aspirin ( $\mathrm{n}=18,270)$ had a relative risk reduction of $22 \%$ (relative risk $0.78,95 \%$ CI $0.71-0.85)$, statins $(n=90,056)$ of $21 \%$ (relative risk $0.79,95 \%$ CI $0.77-0.81$ ) and 
antihypertensives ( $\mathrm{n}=15,527)$ of $21 \%$ (relative risk 0.79 , 95\%CI $0.66-0.95)$.

A negative association of cardiorespiratory fitness and incident stroke, atrial fibrillation and all-cause mortality in primary stroke prevention is well established. [39] However, the role of physical activity and cardiorespiratory fitness in secondary stroke prevention is less clear. A comprehensive meta-analysis ( $n=58$ intervention trials, data included up to 2014, $n=2,797$ participants) investigated the effect of physical activity on vascular mortality after stroke. Only 13 of 2,797 participants died before the end of the intervention and 9 of 1,256 until the end of the follow-up (months). The included participants were at lower risk of death compared with the wider stroke population. However, the inclusion of stroke patients in intervention trials may be biased because of self-selection of physically active participants. [40] A recent meta-analysis (22 RCTs, data included up to 2015, n=2,574 patients after ischaemic stroke/TIA) studied the effects of lifestyle intervention trials, including cardiorespiratory fitness $(n=1,800)$ and combined programmes $(n=222)$ on overall vascular mortality in secondary stroke prevention. A significant reduction in systolic blood pressure (mean difference $-3.6 \mathrm{~mm} \mathrm{Hg}$ ) was achieved in the subgroup where the intervention lasted longer than 4 months and where more than 3 behavioral change techniques were applied. No effect of lifestyle interventions on vascular event rate, mortality, diastolic blood pressure or total cholesterol levels was found, likely because of the small study sample and the short follow-up time of a mean duration of 5.5 months. [41]

An intervention trial on patients with mild stroke/TIA (initially $n=110$ participants, $n=80$ completed the intervention, mean intervention-interval 33 weeks) analyzed the effect of a comprehensive rehabilitation programme consisting of physical activity (2x/week administered by bachelor kinesiologists or 4x/week following patient's choice, supervised by phone), besides an educational programme and diet change on modifiable risk factors. The use of medication was not significantly changed throughout the intervention. The investigators found a significant improvement of cardiorespiratory fitness, total/HDL-cholesterol levels and waist circumference and importantly a highly significant shift $(\mathrm{p}<0.001)$ from low DukeTreadmill-Score (abr. DTS) to higher ones. [42] The DTS was used as an expression of risk of vascular mortality. High DTS scores are associated with lower mortality. The score is calculated taking the duration of physical activity, ECG changes and angina pectoris during the exercise into account.

A meta-analysis of intervention trials (18 RCTs up to 12/2016, $n=930$ participants) showed physical activity as an effective way of reducing resting systolic blood pressure $(\mathrm{p}=0.01)$, fasting glucose $(\mathrm{p}<0.0001)$ and fasting insulin ( $\mathrm{p}=0.03$ ), and increasing HDL-cholesterol levels ( $\mathrm{p}=0.008)$. [43]

The SAMMPRIS trial studied best medical treatment, including a supervised physical activity programme with/without percutaneous angioplasty and stenting of symptomatic intracranial stenosis. [44] The lifestyle modification programme called INTERVENT, previously shown to be effective reducing vascular event rates, took physical activity besides nutrition, stress management and smoking cessation into account. [45] Physical activity was quantified using the Physician-based Assessment and Counseling for Physical Activity (PACE) Self-Report Questionnaire with 3 points corresponding to vigorous or moderate physical activity but not being physically active regularly, 4 points corresponding to moderate physical activity $<5 x /$ week or vigorous physical activity $<3 x /$ week and 6 points corresponding to at least 30 minutes of moderate physical activity a day for at least 5 days a week for the past 6 months or more. [46,47] Physical activity within the target (defined as $\geq 4$ points) had a significant protective effect against myocardial infarction, stroke and vascular death at 3 years (OR 0.5, 95\%CI 0.4-0.7, p<0.05). Other modifiable risk factors such as LDL-/nonHDL-cholesterol levels and systolic blood pressure within the target, also protected against vascular events. 


\section{What about physical activity recommended in stroke survivors in the acute and in}

\section{the rehabilitation phase?}

Physical activity after stroke has the goal of preventing recurrent vascular events and possible post-stroke complications and of improving impaired body functions. The type and quantity of physical activity has to be adapted to individual co-morbidities and to specific residual deficits (e.g. to impaired gait pattern). The current statement of the AHA/ASA from 2014 recommends specific strategies of physical activity according to different goals after stroke: [1]

In the acute phase, evidence is in favour of early mobilization (with few exceptions which we consider at our Bernese Stroke Centre such as e.g. haemodynamic ischaemic stroke, large artery occlusion and persistent penumbra). The goal of mobilization in this phase is to prevent deconditioning, orthostatic intolerance, pneumonia and depression. The intensity of physical activity should be light, with an increase of the HR of 10-20 bpm whilst exercising.

A graded exercise testing under ECG monitoring helps to assess the response of the vascular system and to adequately identify tolerance of physical activity (including optimal intensity and quantity) and thus to adjusted recommended intensity and levels of physical activity after stroke. There are no trials that answer the question of how soon after stroke a graded exercise testing can be safely performed (not done earlier than few weeks after stroke at our Bernese Stroke Centre).

During graded exercise testing, heart rate $>120 \mathrm{bpm}$ or $>70 \%$ of the age-predicted maximal heart rate, systolic blood pressure $>250 \mathrm{mmHg}$ or diastolic blood pressure or $>120 \mathrm{mmHg}$ are criteria recommended for terminating the testing. Clinically relevant ECG abnormalities during testing are ST-segment depression, angina pectoris, ventricular arrhythmia, ventricular tachycardia or bundle branch-block. Although these ECG abnormalities are present in few stroke patients, the frequency of serious adverse events is very low. This may be due to the fact, that stroke residual defects may not allow maximal effort levels.

In the rehabilitation phase a supervised exercise programme is recommended as follows: Aerobic activity such as walking, stationary cycle ergometry, arm ergometry and seated exercises with an intensity of 40-70\% HRmax, 3-5 days/week, 20-60 min sessions (or multiple 10 min sessions) may be performed. The goals of aerobic exercise are to increase walking speed and efficiency, to improve exercise tolerance, to increase independence in activities of daily living (abr. ADL), to improve cognition and to reduce risk factors of stroke.

Muscular strength and indurance exercise helps increasing muscle strength, facilitating to perform leisure time and occupational activities and decreasing the maximal voluntary contraction that a given activity demands. It is suggested to perform 1-3 sets of 10-15 repetitions of 8-10 exercises involving the major muscle groups at $50-80 \%$ of maximum repetition strength, 2-3x/week.

Flexibility exercises such as stretching may prevent contractures, increase the range of movement, improve ADLs and decrease the risk of injury. Static stretches for 10-30 seconds before or after aerobic or strength exercise, 2-3x/week are recommended.

Balance and coordination activities, Tai chi, Yoga, recreational activities using paddles/sport balls to ameliorate hand-eye coordination are said to improve balance, quality of life and mobility and to decrease fear of falling and to increase safety during ADLs. These activities should be used as a complement to aerobic and muscular strength exercises 2-3x/week. 


\section{Conclusions}

Besides improving independence and quality of life, physical activity in stroke survivors improves risk factors such as arterial hypertension, impaired glucose tolerance, overweight, depression, impaired cognitive function, lipid profile and cardiorespiratory fitness. Interestingly, the limiting factors for physical activity after stroke such as impaired balance, falls, fatigue, reduced self-efficacy, depression, reduced cardiorespiratory fitness and impaired cognitive function can be improved through performing individually tailored exercise. Some of these limiting factors such as reduced cardiorespiratory fitness, impaired cognitive function and depression are also independent risk factors for stroke. Implementing efficient secondary prevention measures, including physical activity is challenging, mainly because of reduced awareness or access. However, ambulatory post-stroke programmes including education, addressing risk factors, healthy lifestyle and stroke residual deficits are efficient in reducing risk of recurrent stroke and other vascular events. A supervised exercise programme should be implemented in the rehabilitation phase. Physical activity after stroke appears to be generally safe.

\section{Key messages}

- There are various limiting factors for physical activity after stroke, largely due to post-stroke residual and secondary symptoms, which can be treated by performing physical activity.

- Physical activity is a generally safe and effective intervention in secondary stroke prevention.

- The awareness of the beneficial effect of physical activity after stroke is generally low.

- The current statement of the AHA/ASA from 2014 recommends specific strategies of physical activity according to different goals after stroke.

\section{Korrespondenzadresse}

Adrian Scutelnic

Assistenzarzt

Universitätsklinik für Neurologie

3010 Bern

adrian.scutelnic@insel.ch

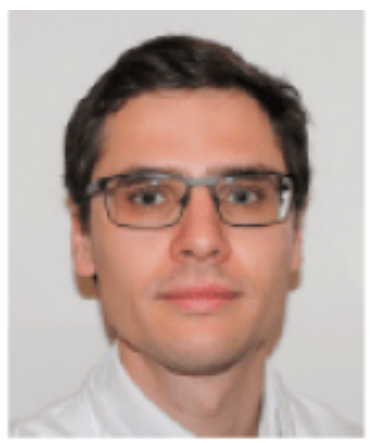


पPD Dr. med. Mirjam R. Heldner, MSc

Oberärztin

Universitätsklinik für Neurologie

3010 Bern

mirjam.heldner@insel.ch

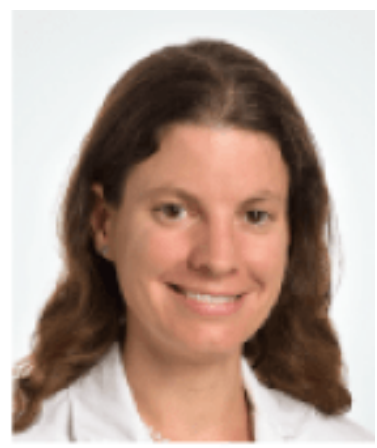

\section{$\square$ References}

1. Billinger SA, Arena R, Bernhardt J, Eng JJ, Franklin BA, Johnson CM et al. American Heart Association Stroke Council; Council on Cardiovascular and Stroke Nursing; Council on Lifestyle and Cardiometabolic Health; Council on Epidemiology and Prevention; Council on Clinical Cardiology. Physical activity and exercise recommendations for stroke survivors: a statement for healthcare professionals from the American Heart Association/American Stroke Association. Stroke. 2014 Aug;45(8):2532-53.

2. Amarenco P, Lavallée PC, Monteiro Tavares L, Labreuche J, Albers GW, Abboud H. Five-Year Risk of Stroke after TIA or Minor Ischemic Stroke. N Engl J Med. 2018 Jun 7;378(23):2182-2190.

3. A Webb, MR Heldner, D Aguiar de Sousa, EC Sandset, G Randall, Y Bejot et al. Availability of secondary prevention services after stroke in Europe: An ESO/SAFE survey of national scientific societies and stroke experts. Eur Stroke J. 2019 Jun;4(2):110-118.

4. Feng W, Hendry RM, Adams RJ. Risk of recurrent stroke, myocardial infarction, or death in hospitalized stroke patients. Neurology. 2010 Feb 16;74(7):588-93.

5. Heldner MR, Li L, Lovett NG, Kubiak MM, Lyons S, Rothwell PM. Long-Term Prognosis of Patients With Transient Ischemic Attack or Stroke and Symptomatic Vascular Disease in Multiple Arterial Beds. Stroke. 2018;49:1639-1646.

6. Hackam DG, Spence JD. Combining multiple approaches for the secondary prevention of vascular events after stroke: a quantitative modeling study. Stroke. 2007 Jun;38(6):1881-5.

7. Boehme C, Toell T, Mayer L, Domig L, Pechlaner R, Willeit K et al. The dimension of preventable stroke in a large representative patient cohort. Neurology. 2019 Oct 31. Epub ahead of print.

8. Hornnes N, Larsen K, Boysen G. Little change of modifiable risk factors 1 year after stroke: a pilot study. Int J Stroke. 2010 Jun;5(3):157-62.

9. Thilarajah S, Mentiplay BF, Bower KJ, Tan D, Pua YH, Williams G et al. Factors Associated With PostStroke Physical Activity: A Systematic Review and Meta-Analysis. Arch Phys Med Rehabil. 2018 Sep; 99(9):1876-1889.

10. Korpershoek C, van der Bijl J, Hafsteinsdóttir TB. Self-efficacy and its influence on recovery of patients with stroke: a systematic review. J Adv Nurs. 2011 Sep;67(9):1876-94.

11. Wali H, Kurdi S, Bilal J, Riaz IB, Bhattacharjee S. Health Behaviors among Stroke Survivors in the United States: A Propensity Score-Matched Study. J Stroke Cerebrovasc Dis. 2018 Aug;27(8):2124-2133.

12. Rand D, Eng JJ, Tang PF, Jeng JS, Hung C. How active are people with stroke?: use of accelerometers to assess physical activity. Stroke. 2009 Jan;40(1):163-8.

13. Pollock A, St George B, Fenton M, Firkins L. Top 10 research priorities relating to life after stroke-consensus from stroke survivors, caregivers, and health professionals. Int J Stroke. 2014;9:313-320. 
14. Bandura, A. (1994). Self-efficacy. In V. S. Ramachaudran (Ed.), Encyclopedia of human behavior (Vol. 4, pp. 71-81). New York: Academic Press. (Reprinted in H. Friedman [Ed.], Encyclopedia of mental health. San Diego: Academic Press, 1998).

15. Chen YK, Qu JF, Xiao WM, Li WY, Weng HY, Li W et al. Poststroke fatigue: risk factors and its effect on functional status and healthrelated quality of life. Int J Stroke. 2015 Jun;10(4):506-12.

16. Staub F, Bogousslavsky J. Fatigue after stroke: a major but neglected issue. Cerebrovasc Dis 2001;12:75-81.

17. Duncan F, Kutlubaev MA, Dennis MS, Greig C, Mead GE. Fatigue after stroke: a systematic review of associations with impaired physical fitness. Int J Stroke. 2012 Feb;7(2):157-62.

18. Choi-Kwon S, Kim JS. Poststroke fatigue: an emerging, critical issue in stroke medicine. Int J Stroke. 2011 Aug;6(4):328-36.

19. Glader EL, Stegmayr B, Asplund K. Poststroke fatigue: a 2-year follow-up study of stroke patients in Sweden. Stroke. 2002 May; 33(5):1327-33.

20. Zedlitz AM, Rietveld TC, Geurts AC, Fasotti L. Cognitive and graded activity training can alleviate persistent fatigue after stroke: a randomized, controlled trial. Stroke. 2012; 43:1046-1051.

21. Dong R, Dong Z, Liu H, Shi F, Du J. Prevalence, Risk Factors, Outcomes, and Treatment of Obstructive Sleep Apnea in Patients with Cerebrovascular Disease: A Systematic Review. J Stroke Cerebrovasc Dis. 2018 Jun; 27(6):1471-1480.

22. Choi-Kwon S, Choi SH, Suh M, Choi S, Cho KH, Nah HW et al. Musculoskeletal and central pain at 1 year post-stroke: associated factors and impact on quality of life. Acta Neurol Scand. 2017 Apr;135(4): 419-425.

23. Hackett ML, Yapa C, Parag V, Anderson CS. Frequency of depression after stroke: a systematic review of observational studies. Stroke. 2005 Jun;36(6):1330-40.

24. Paolucci S, Iosa M, Coiro P, Venturiero V, Savo A, De Angelis D et al. Post-stroke Depression Increases Disability More Than 15\% in Ischemic Stroke Survivors: A Case-Control Study. Front Neurol. 2019 Aug 27;10:926.

25. Eng JJ, Reime B. Physical activity for depressive symptoms in stroke patients: a systematic review and meta-analysis. Clin Rehabil. 2014 Aug;28(8):731-739.

26. Falck RS, Landry GJ, Best JR, Davis JC, Chiu BK, Liu-Ambrose T. Cross-Sectional Relationships of Physical Activity and Sedentary Behavior With Cognitive Function in Older Adults With Probable Mild Cognitive Impairment. Phys Ther. 2017 Oct 1;97(10):975-984.

27. Jin YP, Di Legge S, Ostbye T, Feightner JW, Hachinski V. The reciprocal risks of stroke and cognitive impairment in an elderly population. Alzheimers Dement. 2006;2:171-178.

28. Cumming TB, Tyedin K, Churilov L, Morris ME, Bernhardt J. The effect of physical activity on cognitive function after stroke: a systematic review. Int Psychogeriatr. 2012;24:557.

29. Batchelor FA, Mackintosh SF, Said CM, Hill KD. Falls after stroke. Int J Stroke. 2012 Aug;7(6):482-90.

30. Duncan PW, Sullivan KJ, Behrman AL, Azen SP, Wu SS, Nadeau SE et al. Body-weight supported treadmill rehabilitation after stroke. N Engl J Med2011;364:2026-36.

31. Marigold DS, Eng JJ, Dawson AS, Inglis JT, Harris JE, Gylfadottir S. Exercise leads to faster postural reflexes, improved balance and mobility, and fewer falls in older persons with chronic stroke. J Am Geriatr Soc 2005;53:416-23.

32. Kim CM, Eng JJ. Magnitude and pattern of 3D kinematic and kinetic gait profiles in persons with stroke: relationship to walking speed. Gait Posture. 2004 Oct;20(2):140-6.

33. Kramer S, Johnson L, Bernhardt J, Cumming T. Energy Expenditure and Cost During Walking After Stroke: A Systematic Review. Arch Phys Med Rehabil. 2016 Apr;97(4):619-632.e1. 
34. Banks G, Bernhardt J, Churilov L, Cummin TB. Physical activity Preferences Are Different after Stroke. Stroke Research and Treatment, 2012.

35. Marzolini S, Balitsky A, Jagroop D, Corbett D, Brooks D, Grace SL et al. Factors Affecting Attendance at an Adapted Cardiac Rehabilitation Physical activity Program for Individuals with Mobility Deficits Poststroke. J Stroke Cerebrovasc Dis. 2016 Jan;25(1):87-94.

36. Billinger SA, Coughenour E, Mackay-Lyons MJ, Ivey FM. Reduced cardiorespiratory fitness after stroke: biological consequences and physical activity-induced adaptations. Stroke Res Treat. 2012; 2012:959120.

37. MacKay-Lyons MJ, Makrides L. Physical activity capacity early after stroke. Archives of Physical Medicine and Rehabilitation. 2002;83(12):1697-1702.

38. Luo L, Meng H, Wang Z, Zhu S, Yuan S, Wang Y et al. Effect of high-intensity physical activity on cardiorespiratory fitness in stroke survivors: A systematic review and meta-analysis. Ann Phys Rehabil Med. 2019 Aug 26.

39. Hussain N, Gersh BJ, Gonzalez Carta K, Sydó N, Lopez-Jimenez F, Kopecky SL et al. Impact of Cardiorespiratory Fitness on Frequency of Atrial Fibrillation, Stroke, and All-Cause Mortality. Am J Cardiol. 2018 Jan 1;121(1):41-49.

40. Saunders DH, Sanderson M, Hayes S, Kilrane M, Greig CA, Brazzelli M et al: Physical fitness training for stroke patients. Cochrane Database Syst Rev. 2016 Mar 24;3:CD003316.

41. Deijle IA, Van Schaik SM, Van Wegen EE, Weinstein HC, Kwakkel G, Van den Berg-Vos RM. Lifestyle Interventions to Prevent Cardiovascular Events After Stroke and Transient Ischemic Attack: Systematic Review and Meta-Analysis. Stroke. 2017 Jan;48(1):174-179.

42. Prior PL, Hachinski V, Unsworth K, Chan R, Mytka S, O’Callaghan C et al. Comprehensive Cardiac Rehabilitation for Secondary Prevention After Transient Ischemic Attack or Mild Stroke: I: Feasibility and Risk Factors. Stroke 2011;42:3207-13. 10.1161/STROKEAHA.111. 620187

43. D'Isabella NT, Shkredova DA, Richardson JA, Tang A. Effects of physical activity on cardiovascular risk factors following stroke or transient ischemic attack: a systematic review and meta-analysis. Clin Rehabil. 2017 Dec;31(12):1561-1572.

44. Derdeyn CP, Chimowitz MI, Lynn MJ, Fiorella D, Turan TN, Janis LS et al. Stenting and Aggressive Medical Management for Preventing Recurrent Stroke in Intracranial Stenosis Trial Investigators. Lancet. 2014 Jan 25;383(9914):333-41.

45. https://www.interventhealth.com, last access 02.11.19

46. Turan TN, Linn MJ, Nizam A, Lane B, Egan BM, Le NA et al. Rationale, Design, and Implementation of Aggressive Risk Factor Management in the SAMMPRIS Trial. Circ Cardiovasc Qual Outcomes. 2012 September 1;5(5):e51-e60.

47. Turan TN, Nizam A, Lynn MJ, Egan BM, Le NA, Lopes-Virella MF et al. Relationship between risk factor control and vascular events in the SAMMPRIS trial. Neurology. 2017 Jan 24;88(4):379-385. 